\title{
Paradigmenwechsel im Strafrecht: Die Notwendigkeit einer „Neuen Strafrechtswissenschaft"?
}

- Überlegungen zur 35. Strafrechtslehrertagung in Zürich -

Marc Engelhart, Max-Planck-Institut für ausländisches und internationales Strafrecht, Freiburg i.Br."

\section{A. Einleitung}

In vielen Diskussionsrunden herrschte Einigkeit: Der Titel „Strafrechtliche Probleme des Finanzmarktes “ der 35. Strafrechtslehrertagung in Zürich, der an die Behandlung einzelner Besonderheiten eines strafrechtlichen Spezialgebiets denken ließ, verdeckte, dass es tatsächlich um grundsätzliche Fragen des Strafrechts und nicht allein des Wirtschaftsstrafrechts ging. Mehr noch: Im Kern ging es um eine Neuausrichtung des Strafrechts und eine Neujustierung der Strafrechtswissenschaft. Passend wäre daher gewesen: ,Paradigmenwechsel im Strafrecht: Die Notwendigkeit einer „Neuen Strafrechtswissenschaft"??.

Woran lassen sich nun diese weitreichenden Änderungen festmachen und worin liegen sie? So einfach diese Fragen sind, so schwierig ist ihre Beantwortung. Dies zeigten die Diskussionen im und außerhalb des Plenums. Denn bislang hat man es nur mit zwei Phänomenen zu tun: Compliance und Privatisierung. Beschrieben haben sie insbesondere Rotsch („Compliance und Strafrecht - Bedeutung, Fragen, Perspektiven“), Kölbel („Criminal Compliance - ein Missverständnis des Strafrechts?“), Bung („Grundlagenprobleme der Privatisierung von Sanktions- und Präventionsaufgaben“) und Zerbes („Unternehmensinterne Untersuchungen“) in ihren Beiträgen. Die genauen Konturen dieser Phänomene sind dagegen nicht klar, ihre Entwicklung ist noch im Fluss und ihr Ziel, auf das sie sich zubewegen, ist noch im Ungewissen. Das macht es so schwierig, zu bestimmen, ob es sich um eine harmlose Abwandlung von Altbekanntem handelt oder aber doch eher um den Wolf im Schafspelz. Gerade bei der Diskussion zu Compliance herrschte somit - kaum verwunderlich - Uneinigkeit, ob es sich um „alten Wein in neuen Schläuchen“ handele oder um echte Neuerungen. Die folgenden Überlegungen, die in diesem Rahmen selektiv und kursorisch bleiben müssen, möchten verdeutlichen, warum mit den beiden Themen die Rolle des Strafrechts im Wandel begriffen ist (dazu B.), der auch ein neues Verständnis der Strafrechtswissenschaft erfordert (C.).

\section{B. Die Veränderungen im Strafrecht}

Das Strafrecht hat im Kern klassischerweise strafwürdiges und strafbedürftiges Verhalten und dessen Bestrafung zum Gegenstand. Mit den Themen Compliance und Privatisierung gewinnen nunmehr aber übergreifende präventive Aspekte, private

* Dr. Marc Engelhart leitet am Max-Planck-Institut für ausländisches und internationales Strafrecht das Referat Wirtschaftskriminalität und Wirtschaftsstrafrecht. 
Regelsetzung und -durchsetzung und die Internationalisierung einen ganz neuen Stellenwert.

\section{Prävention}

Mit Compliance öffnet sich das Strafrecht der Prävention. Präventive Elemente sind dem Strafrecht nicht fremd, aber das Maß an Prävention durch konkrete Maßnahmen, das Bestandteil „guter" Compliance ist, schon. Compliance erfordert aktive Maßnahmen von Unternehmen, um strafrechtliche Verstöße von Unternehmensangehörigen zu verhindern. Angesprochen sind aber nicht die Unternehmen allein, sondern ebenfalls Individuen und die ihnen obliegenden Pflichten. Auch ist die Frage nicht auf die (in der Praxis am relevantesten) Unternehmen begrenzt, sondern bezieht sich auf jede verfestigte Organisation. Zentral für die Prävention sind allein effektive Compliance-Maßnahmen. Anerkanntermaßen genügt dabei nicht ein Papier-Kodex mit Vorgaben, wie man sich verhalten soll, sondern es bedarf zahlreicher Anreiz- und Kontrollmechanismen auf allen Ebenen (des Unternehmens). Entsprechende Pflichten lassen sich aber weder im bisherigen Strafrecht noch im Ordnungswidrigkeitenrecht finden. $\mathbb{S} 13 \mathrm{StGB}$ mit seinen Garantenpflichten ist, auch wenn man diesen weit versteht und eine umfassende Geschäftsherrenhaftung akzeptiert, allenfalls für einzelne Pflichten maßgeblich, konstituiert jedoch nicht das Erfordernis eines umfangreichen und auf Dauer angelegte Compliance-Programms. Der Idee von Compliance noch am nächsten kommt $\mathbb{S} 130 \mathrm{OWiG}$ mit der Sanktionierung der Aufsichtspflichtverletzung. Aber auch der Wortlaut von $\$ 130 \mathrm{OWiG}$ spricht nicht von komplexen Präventivstrukturen, die für eine angemessene Aufsicht erwartet werden, und nennt schon gar keine konkreten Vorgaben. Möglicherweise kann sich aufgrund der Compliance-Entwicklung eine Auslegung, die ein Compliance-Programm als erforderlich ansieht, durchsetzen. Dies wäre jedoch erst Ergebnis und nicht Ausgangspunkt der Compliance-Diskussion. Bleibt festzuhalten, dass mit Compliance die Vermeidung von Rechtsverstößen und nicht erst der Rechtsverstoß und dessen Behandlung in den strafrechtlichen Fokus rückt. „Vermeidung“ ist dabei wie von Rotsch beschrieben weit zu verstehen, es geht schon um die Vermeidung des Verdachts einer Straftat, ja um die Vermeidung ungewollter Ermessensausübung. Dieser Ansatz der maximalen Risikoreduzierung lenkt den Blick von der einzuhaltenden Regel auf die tatsächlichen Umstände, die zum Regelbruch führen und wie sie effektiv (ggf. auch durch regulative Umsetzung) minimiert werden können.

Ein Problem von Compliance ist ihre Komplexität. Es ist eine Querschnittsmaterie, die nicht auf das Strafrecht (selbst bei weitem Verständnis und Einbeziehung des Ordnungswidrigkeitenrechts) beschränkt ist. Criminal Compliance mag der wichtigste Bereich von Compliance sein, er ist jedoch nur einer von mehreren. Compliance, wie dies Rotsch definiert, ist Regelkonformität, und damit kann die „Regel“ aus jedem Rechtsbereich stammen und das Zivil- wie auch das Verwaltungsrecht 
umfassen. Zum zwingenden Recht kommt noch das „soft law“ hinzu (siehe unten II.). Die vorzunehmenden Compliance-Maßnahmen zur Prävention von Regelbrüchen sind aber für zwingende wie freiwillige Regelungen grundsätzlich gleich, allenfalls erfordern spezifische, insbesondere hoch regulierte Bereiche (wie die Prävention von Insiderhandel im Bankenbereich) besondere Maßnahmen (z.B. chinese walls). Das Strafrecht lässt sich damit nicht isoliert betrachten, sondern muss andere (Rechts-)Bereiche von vornherein mit in den Blick nehmen.

\section{Privatisierung}

Compliance bedeutet zudem die Öffnung des Strafrechts für eine private Regelsetzung und-durchsetzung, die den staatlichen Bereich ergänzt oder überlagert. Im Bereich der Regelsetzung treten neben die „offiziellen“ Normen private Vorgaben, die staatliche wiederholen, konkretisieren und erweitern. Sie können aber wie bei der Umsetzung ethischer Belange und einer bestimmten „corporate culture“ auch ganz eigenständigen Charakter annehmen, wenn sie weit über das Recht als ethisches „Minimum “ hinausgehen. Gerade in Compliance-Programmen spielen ethische Normen eine große Rolle, da sie Einstellungen und Werte prägen und damit die Basis für rechtmäßiges Verhalten legen. Die Umsetzung dieser Mischung an privaten und staatlichen Vorgaben in Compliance-Programmen zeigt, dass es nicht - wie häufig angenommen - allein um unternehmensinterne, der Privatautonomie der Unternehmen überlassene, Systeme geht. Vielmehr finden sich verschiedenste Möglichkeiten staatlicher Einflussnahme: Diese können unverbindlich sein, wie die Muster-Compliance-Programme amerikanischer Bundesbehörden. Diese können stärkere rechtliche Relevanz haben wie die Vorgaben der deutschen Bundesanstalt für Finanzdienstleistungen (BaFin) in den sog. MaComp zu Compliance-Programmen im Wertpapierhandelsrecht ${ }^{1}$ oder die Berücksichtigung von Compliance-Programmen als Strafmilderungsgrund in den amerikanischen Strafzumessungsrichtlinien für Unternehmen. Schließlich können sie auch bis zum Ausschluss der Verantwortlichkeit des Unternehmens reichen wie etwa im britischen UK Bribery Act 2010, wenn Mitarbeiter bei Vorliegen eines effektiven Compliance-Programms Korruptionstaten begehen. Erste Ansätze in diese Richtung finden sich nun auch im Bankensektor in Deutschland, wo der neue $\mathbb{5} 54 \mathrm{a}$ KWG eine Strafbarkeit vorsieht, wenn ein mangelhaftes Risk Management System zu einer Bestandsgefährdung des Instituts führt. ${ }^{2}$

1 BaFin, Rundschreiben 4/2010 (WA) - Mindestanforderungen an die Compliance-Funktion und die weiteren Verhaltens-, Organisations- und Transparenzpflichten nach $\mathbb{S} \$ 31 \mathrm{ff}$. WpHG für Wertpapierdienstleistungsunternehmen (MaComp), Stand: 30. November 2012.

2 Vgl. $\$ 54 \mathrm{a}$ iVm. $\$ 25 \mathrm{c} \mathrm{KWG}$ in der Fassung des Gesetzes zur Abschirmung von Risiken und zur Planung der Sanierung und Abwicklung von Kreditinstituten und Finanzgruppen, BT-Drs. 17/12601, 17/13523. Das Gesetz ist am 17.5.2013 vom Bundestag beschlossen worden, der Bundesrat hat den Vermittlungsausschuss nicht angerufen, so dass die Vorschriften am 2.1.2014 in Kraft treten werden. 
Diese Mischung privater und staatlicher Ansätze macht deutlich, dass es vor allem um regulierte Selbstregulierung geht. Dabei stellt sich aus regulatorischer Sicht die Frage, welche Mechanismen der Staat hier nutzen kann. Kölbel zeigt in seinem Referat, dass das bestehende deutsche Rechtsumfeld Unternehmen wenig Anreize zur effektiven Gestaltung von Compliance-Programmen bietet. Ein wesentlicher Grund liegt darin, dass die Frage, wie eine bessere Regulierung der Selbstregulierung zu erreichen ist, gerade aus strafrechtlicher Sicht noch unterthematisiert ist und die Diskussion auf wenig grundsätzlicher Ebene geführt wird. Die Überlegung von Kölbel, den Weg einer eigenständigen Sanktionierung des Unterlassens von Compliance zu beschreiten, ist eine von zahlreichen Möglichkeiten. Doch bevor ein solcher Schritt gemacht wird, sollten zunächst einmal die möglichen „weichen“ und „harten“ Steuerungsmechanismen eruiert und evaluiert werden.

Neben der Regelsetzung erfolgt auch die Durchsetzung von Regeln nur teilweise staatlich. Ein wesentlicher Teil ist privatisiert. Insbesondere Kontrollen werden in den Unternehmen zunehmend intensiviert, vielfach erfolgen sie aufgrund von Compliance-Programmen regelmäßig, systematisch und standardisiert. Dabei wird stark auf transparentes Agieren der Mitarbeiter und eine entsprechende gegenseitige Kontrolle durch Kollegen und das Management gesetzt. Dieser Mechanismus der Transparenz, gerade wenn die Unternehmensführung rechtskonformes Verhalten positiv vorlebt und bestärkt, wirkt auf die Mitarbeiter ein und kann deren Verhalten beeinflussen. Der Ansatz von Bung, Compliance als „panoptisches Regieren“ im Sinne Foucaults zu begreifen, das beim Individuum zur Vermeidung von Straftaten führt, da diese um die Sichtbarkeit ihres Tuns wissen, ist daher - auch bei aller Vorsicht, die walten sollte, wenn man die Theorien Foucaults auf diese Konstellation überträgt - nicht fernliegend. Denn Foucault stellt zu Recht heraus, dass Regieren als Machtausübung vor allem „Führung “ bedeutet, ${ }^{3}$ und genau darum geht es bei Compliance: Sie soll als vom Unternehmen vorgegebener Rahmen zu rechtmäßigem Verhalten führen.

Zur standardisierten Regeleinhaltung durch Compliance-Maßnahmen kommen private interne Ermittlungen („Internal Investigations“) hinzu. Seit der umfangreichen Aufklärung der Korruptionsfälle bei Siemens sind derartige Ermittlungen auch einer breiteren Öffentlichkeit bekannt. Als repressive Seite können sie eng mit Compliance verwoben sein und werden daher auch oft als Compliance-Ermittlungen bezeichnet. Abstrakte und formalisierte Vorgaben zum Ablauf solcher Ermittlungen in Compliance-Programmen schaffen dabei ein privates Sanktionsverfahrensrecht. Wie der Beitrag von Zerbes zeigte, sind diese Ermittlungen dabei in vielfacher Weise mit staatlichen verschränkt oder haben auf diese Auswirkungen. Dies wirft nicht nur ganz neue Fragen zur Zulässigkeit und zum Vorgehen solch „privater“ Ermittlungen,

3 Foucault, Dits et Écrits. Schriften, Bd. 4, Frankfurt a. M. 2005, S. 286 f. 
sondern auch zum Ablauf des parallelen oder als Annex betriebenen staatlichen Ermittlungs- und Sanktionsverfahren und etwa der Sanktionsbemessung (Kosten interner Ermittlungen als Abzugsposten?) auf. Vor allem sind jedoch die anzulegenden Schutzstandards unklar. Die an den Staat adressierte StPO als „magna charta“ des Verbrechers stößt hier an ihre Grenzen und droht durch die unter dem Primat der Privatautonomie des Unternehmens stattfindenden Untersuchungen ausgehöhlt zu werden.

\section{Internationalisierung}

Schließlich wird das Strafrecht durch Compliance in hohem Maße internationalisiert, und zwar stärker, als es bislang der Fall ist. Compliance zielt auf die Einhaltung aller für ein Unternehmen oder einen Mitarbeiter relevanten Normen. Dies sind natürlich in erster Linie deutsche Vorgaben, aber eben auch alle ausländischen, die aufgrund eines Auslandsbezugs gelten. Besonders prominente Beispiele finden sich in der Korruptionsbekämpfung: Der amerikanische Foreign Corrupt Practices Act oder der britische UK Bribery Act 2010 gelten weltweit. Damit sind Fragen der Reichweite des angloamerikanischen Konzepts der „conspiracy“ nicht allein mehr Ausdruck eines gewissen auslandsrechtlichen Interesses, sondern originäres Problem eines im common law tätigen Unternehmens, das auch in Deutschland die Einhaltung dieser Vorgaben sicherzustellen hat. Hinzu kommen die Regelungen internationaler Institutionen wie die UN Global Compact Initiative der Vereinten Nationen oder die OECD-Leitsätze für multinationale Unternehmen, zu deren Einhaltung sich zahlreiche Unternehmen freiwillig verpflichtet haben. Damit ist Compliance, wie von Rotsch angeführt, "globalisiertes Strafrecht“, vor allem aber ein zunehmend standardisiertes und Nationalgrenzen überschreitendes Präventionsinstrument. Friktionen oder gar Widersprüche nationaler Strafrechtsregelungen, die im schlimmsten Fall in dem einen Land das gebieten, was im anderen verboten ist, stellen hierbei die größte Schwierigkeit dar und verlangen nach einer Harmonisierung.

\section{Zwischenfazit}

Compliance zeigt deutlich, dass sich die klassischen Grenzen des nationalen Strafrechts verschieben und dass das staatliche System der Sozialkontrolle durch das Hinzutreten privater Akteure maßgeblich verändert wird. Insbesondere der Schwerpunkt auf Präventionsmaßnahmen und die Frage nach ihrer strafrechtlichen Relevanz verlagert den Blick vom Strafrecht als repressivem Instrument auf die Möglichkeiten eines präventiv orientierten Steuerungsmechanismus. Da in Deutschland Compliance als Instrument regulierter Selbstregulierung nur ansatzweise Eingang in staatliche Überlegungen gefunden hat, ist die Tragweite dieser Schwerpunktverlagerung noch nicht abzusehen. Dieser Übergang zum Einsatz (auch) des Strafrechts als Mittel des Gewährleistungsstaates, in dem mehr die Interaktion mit dem Bürger als die hoheit- 
liche Über-/Unterordnungsebene betont wird, kann aber sicherlich bereits heute als Paradigmenwechsel bezeichnet werden.

\section{Herausforderungen für die Strafrechtswissenschaft}

Die vorgenannten Veränderungen des Strafrechts stellen die Strafrechtswissenschaft vor neue Herausforderungen. Auch wenn eine umfassende Analyse im vorliegenden Rahmen nicht möglich ist, ${ }^{4}$ so sollen doch drei zentrale Aspekte, die zu einer Verlagerung der wissenschaftlichen Schwerpunkte führen, kurz thematisiert werden: das Strafrecht als Teil sozialer Konfliktlösung, die Wirklichkeitsanalyse und die Internationalisierung.

In ihrem Grußwort zur Strafrechtslehrertagung sah die schweizerische Bundesrätin Sommargu die Rolle des Strafrechts in der eines Konfliktbewältigungsmechanismus. In die gleiche Richtung zielte die Betrachtung von Kölbel, bei Compliance gehe es um die Frage des „Governing through Crime“. Diese Sichtweise zeigt zum einen die hohen gesellschaftlichen (und teilweise vielleicht kaum einlösbaren) Erwartungen an das Strafrecht, macht aber zum anderen auch deutlich, dass das Strafrecht verstärkt als Teil einer umfassenderen Strategie zur Bewältigung bestimmter sozialer Konflikte gesehen werden sollte. Das Strafrecht ist integrativer Bestandteil eines Gesamtkonzepts möglicher staatlicher und gesellschaftlicher Lösungen. Dies erfordert, den Schwerpunkt der Strafrechtwissenschaft von einer dogmatisch orientierten Strukturierungs- und Interpretationswissenschaft zu einer mehr rechtsetzungsorientierten Handlungs- und Entscheidungswissenschaft zu verlagern. Dazu bedarf es eines breiten Ansatzes, den einer eigenständigen „Sanktionsrechtswissenschaft“, die neben Strafen (einschließlich Geldbußen) auch andere ahndende Maßnahmen sowie weitere staatliche und private Steuerungsmechanismen sowie ihre Mischformen der regulierten Selbstregulierung in den Blick nimmt. In die Betrachtung sind dabei aufgrund vielfacher Verschränkungen und Auswirkungen nicht allein materielle Rege-

4 Zur Diskussion über die Herausforderungen der heutigen Strafrechtswissenschaft siehe insbes. die Beiträge in Eser (Hrsg.), Die deutsche Strafrechtswissenschaft vor der Jahrtausendwende: Rückbesinnung und Ausblick, München 2000 sowie Frisch, Gesellschaftlicher Wandel als formende Kraft und als Herausforderung des Strafrechts, in: Müller-Dietz/Müller/Kunz u.a. (Hrsg.), Festschrift für Heike Jung, Baden-Baden 2007, S. 189 ff.; Schünemann, Kritische Anmerkungen zur geistigen Situation der deutschen Strafrechtswissenschaft, GA 1995, S. 201 ff.; ders., Die deutsche Strafrechtswissenschaft nach der Jahrtausendwende, GA 2001, S. 205 ff.; ders., Standpunkte der deutschen Strafrechtslehrer zu den Zukunftsperspektiven der Rechtswissenschaft und der akademischen juristischen Ausbildung in Deutschland, ZIS 2012, S. 302 ff.; Weigend, Wohin bewegt sich das Strafrecht? in: Freund/Murmann/Bloy/Perron (Hrsg.), Grundlagen und Dogmatik des gesamten Strafrechtssystems. Festschrift für Wolfgang Frisch zum 70. Geburtstag, Berlin 2013, S. 17 ff.; siehe auch Sieber, Grenzen des Strafrechts, ZStW 119 (2007), S. 1 ff.; ders., Rechtliche Ordnung in einer globalen Welt, Rechtstheorie 41 (2010), S. 151 ff. Zum Anpassungsbedarf der Strafrechtsdogmatik siehe bspw. Kindhäuser, Die deutsche Strafrechtsdogmatik zwischen Anpassung und Selbstbehauptung - Grenzkontrolle der Kriminalpolitik durch die Dogmatik?, ZStW 121 (2009), S. 954 ff.; Landauer, Die deutsche Strafrechtsdogmatik zwischen Anpassung und Selbstbehauptung - Grenzkontrolle der Kriminalpolitik durch die Dogmatik?, ZStW 121 (2009), 965 ff. sowie Gracia Martín, Über die notwendige Modernisierung des Strafrechts in der deutschen und spanischen Doktrin, GA 2010, S. 323 ff. 
lungen, sondern gleichermaßen prozessuale einzubeziehen, um eine (bessere) Abstimmung beider Bereiche zu erreichen. Eine derartige Herangehensweise ist originär rechtsgebietsübergreifend, da sie, ausgehend von verfassungsrechtlichen Legitimationsfragen $^{5}$, neben strafrechtlichen Ansätzen beispielsweise die Steuerungsdiskussion des öffentlichen Rechts oder die Corporate-Governance-Diskussion des Zivilrechts zu berücksichtigen hat. Für diesen Blick aufs Ganze ist eine neue Art von Generalist, der methodisch offen ist ${ }^{6}$, notwendig.

Eine auf die Lösung sozialer Probleme abzielende Betrachtung bedarf in besonderem Maße der Kenntnis der Wirklichkeit, also einer Wirklichkeits- oder Realbereichsanalyse $e^{7}$. Nur wenn ein Problem faktisch ausreichend beschrieben ist, lässt es sich normativ auf Tatbestands- wie Rechtsfolgenseite entsprechend erfassen. Gefragt ist daher ein theoretisches Denken auf empirischer Basis und mit empirischer Überprüfung. Im Rahmen einer Wirkungs- und Folgenanalyse sind dabei die Auswirkungen der Regelung (jenseits einer möglichen Abschreckungswirkung) stärker als bisher in den Blick zu nehmen. Dies gilt insbesondere für die Einbeziehung nichtstrafrechtlicher Maßnahmen, um abzuschätzen, ob diese effektiver, effizienter und gegebenenfalls gegenüber dem Strafrecht vorzugswürdig sind. Derartige Realanalysen und Abschätzungen bedingen einen interdisziplinären Ansatz, der beispielsweise die Soziologie, die Psychologie, die Wirtschafts- und Politikwissenschaften miteinbezieht. Mit der Kriminologie besteht in vielerlei Hinsicht bereits ein derart übergreifendes und auch etabliertes Forschungsfeld. Deren Methodik und Erkenntnisse sollten, wie ursprünglich im Ansatz einer „gesamten Strafrechtswissenschaft“ intendiert, originär in die strafrechtliche Forschung miteingebunden, weiterentwickelt und gestärkt werden. ${ }^{8}$

Letztlich bedarf es auch einer stärkeren Berücksichtigung der ausländischen und internationalen Entwicklung in der Forschung, und damit einer weiteren Vertiefung des seit gut zwei Jahrzehnten stattfindenden Diskurses. ${ }^{9}$ Der Versuch, ComplianceProgramme für alle in Frage kommenden Regelungen zu entwerfen, bedarf einer Analyse und Bewertung des in- wie ausländischen (Straf-)rechts, um die Gemein-

5 Vgl. zum unzureichenden Rekurs der Strafrechtswissenschaft auf verfassungsrechtliche Aspekte etwa Stuckenberg, Grundrechtsdogmatik statt Rechtsgutslehre, GA 2011, S. 653 ff.

$6 \mathrm{Zu}$ diesem wichtigen Aspekt siehe Vogel, Strafrecht und Strafrechtswissenschaft im internationalen und europäischen Rechtsraum, JZ 2012, S. 25 (30) sowie bereits Kübl, Europäisierung der Strafrechtswissenschaft, ZStW 109 (1997), S. 777 (801).

7 Vgl. zur öffentlich-rechtlichen Perspektive Voßkuble, Neue Verwaltungsrechtswissenschaft, in: Hoffmann-Riem/Schmidt-Aßmann/Voßkuhle (Hrsg.), Grundlagen des Verwaltungsrechts, Bd. I, Methoden - Maßstäbe - Aufgaben - Organisation, 2. Aufl. München 2012, 11 Rn. 29.

8 Für eine Stärkung der Kriminologie - wenn auch als eigenständiges Fach - siehe Albrecht/Quensel/ Sessar (Hrsg.), Freiburger Memorandum. Zur Lage der Kriminologie in Deutschland. Freiburg i. Br. 2012.

9 Vgl. dazu auch Hirsch, Internationalisierung des Strafrechts und Strafrechtswissenschaft, ZStW 116 (2004), S. 835; Vogel, Strafrechtswissenschaft (Fn. ), JZ 2012, S. 25 sowie insgesamt zur Rechtswissenschaft $v$. Bogdandy, Deutsche Rechtswissenschaft im europäischen Rechtsraum, JZ 2011, S. 1. 
samkeiten und Unterschiede herauszuarbeiten. Somit kommt der Rechtsvergleichung ein hoher Stellenwert zu. Unterschiede nationaler Regelungen lassen dabei leicht den Ruf nach einer Harmonisierung erschallen. Hier gilt es zu analysieren, ob das deutsche Recht verzichtbare Vorgaben enthält oder ob die deutsche Regelung aufgrund überzeugender Argumente nicht preisgegeben werden sollte („deutsche Strafrechtskultur “10). Da viele Vorgaben, verbindlich etwa über die EU oder unverbindlich etwa über Empfehlungen der OECD, inzwischen auf supranationaler Ebene getroffen werden, ist derartiges Wissen unentbehrlich. ${ }^{11}$ Hier gilt es im internationalen Markt der Ideen auch die deutsche verstärkt einzubringen und zu etablieren. Mit überzeugenden Konzepten, von denen das deutsche Recht und insbesondere die hochentwickelte und ausdifferenzierte deutsche Strafrechtswissenschaft viele zu bieten hat, besteht immerhin die Chance, dass sich der beste Ansatz durchsetzt und nicht allein der, der numerisch gesehen schon in den meisten Ländern gesetzlich vorhanden ist. Voraussetzung für die Teilnahme am internationalen Wissenschaftsmarkt, für die internationale Anschlussfähigkeit, ist die Verwendung einer verkehrsfähigen Sprache. Diese ist nur in Bezug auf wenige Länder - und dort zumeist beschränkt auf einige Personen - Deutsch, sonst aber vor allem Englisch, zum Teil auch noch Spanisch. Nur wenn die deutschen Ideen in der entsprechenden „lingua franca“ vorhanden sind, werden sie wahrgenommen werden.

\section{Fazit}

Die Phänomene Compliance und Privatisierung zeigen nicht nur die Veränderungen des Strafrechts, sondern auch die Notwendigkeit einer neuen Schwerpunktsetzung in der Strafrechtswissenschaft. Im Verwaltungsrecht haben ähnliche Veränderungen - die „Krise des regulativen Rechts“ aufgrund gesellschaftlicher und technischer Wandlungen mit Reformansätzen für die Verwaltung und des von ihr anzuwendenden Rechts - dazu geführt, dass sich eine „Neue Verwaltungsrechtswissenschaft“" etabliert hat. ${ }^{12}$ Es scheint daher auch die Zeit für eine „Neue Strafrechtswissenschaft" gekommen zu sein, die sich thematisch und methodisch breiter und internationaler aufstellt.

10 Vgl. zur Thematik Vogel, Transkulturelles Strafrecht, GA 2010, S. 1 ff.

11 Schünemann, Standpunkte (Fn. ), ZIS 2012, S. 302 sieht die wichtigste Herausforderung in der Einflussnahme auf das Strafrecht der EU.

12 Eingehend Voßkuble, Neue Verwaltungsrechtswissenschaft, in: Hoffmann-Riem/Schmidt-Aßmann/ Voßkuhle (Hrsg.), Grundlagen (Fn. ), $\$ 1$ Rn. 16 ff. 